\section{Parallel pain pathways}

Pain consists of a sensory component (the detection and transmission of painful stimuli) and an affective component (the processing of emotional and behavioural responses). Writing in Neuron, Braz and colleagues show that different populations of nociceptors - neurons that sense painful stimuli - might engage parallel ascending pathways that target limbic (affective) and motor regions of the brain.

Nociceptors, which are located in the dorsal root ganglia and trigeminal ganglia, have a peripheral axonal branch that receives sensory information from the peripheral tissues, and a central branch that transmits signals to the spinal cord, from where they are relayed to the brain. There are two main types of nociceptor: peptidergic nociceptors, which express neuropeptides and terminate in the most superficial laminae of the dorsal horn, targeting lamina I projection neurons and interneurons of outer lamina II; and nonpeptidergic nociceptors, which target interneurons of inner lamina II.

In this study, Braz et al. looked at nonpeptidergic circuits by genetically targeting a transneuronal tract tracer, wheat germ agglutinin, to a subpopulation of nonpeptidergic nociceptors that express the tetrodotoxin-resistant sodium channel Nav1.8. They found that the major ascending circuits begin with the interneurons of inner lamina II, which are targeted by nonpeptidergic nociceptors. These interneurons are connected

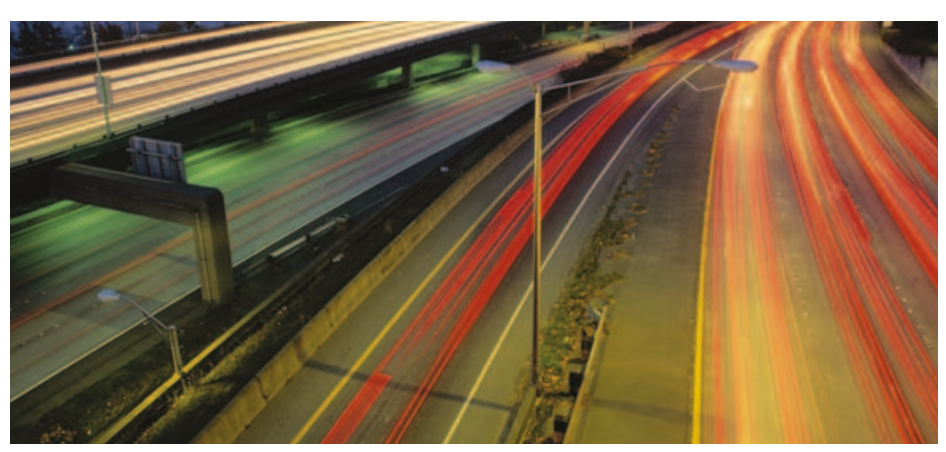

to lamina $\mathrm{V}$ projection neurons, which, in turn, target neurons in the amygdala, hypothalamus and bed nucleus of the stria terminalis - limbic regions of the brain that are also targeted by peptidergic nociceptors through a polysynaptic pathway involving the parabrachial nuclei of the dorsolateral pons.

In addition, there is a remarkably concentrated projection to the globus pallidus, an area of the brain rarely considered when discussing ascending nociceptive circuits. As the globus pallidus is involved in regulating motor functions, this newly discovered pain target might explain why noxious stimuli almost always induce changes in motor behaviours as well as evoking perception and the emotional responses of pain, which are mediated by limbic regions of the brain.

Therefore, nonpeptidergic nociceptors might give rise to a parallel, independent pain pathway that targets predominantly motor and limbic centres. It will be interesting to determine how peptidergic and nonpeptidergic circuits interact in the brain and whether they converge on the same limbic subregions. Jane Qiu

\section{(1) References and links}

ORIGINAL RESEARCH PAPER Braz, J. M. et al. Parallel "pain" pathways arise from subpopulations of primary afferent nociceptor. Neuron $\mathbf{4 7}$ 787-793 (2005)

FURTHER READING Craig, A. D. Pain

mechanisms: labeled lines versus convergence in central processing. Annu. Rev. Neurosci. 26, 1-30 (2003)

\section{WEB SITE}

Basbaum's laboratory: http://www.ucsf.edu/ pibs/faculty/basbaum.html

\section{IN BRIEF}

\section{NEUROIMAGING}

Imaging input and output of neocortical networks in vivo.

Kerr, J. N. D., Greenberg, D. \& Helmchen, F. Proc. Natl Acad. Sci. USA 102, 14063-14068 (2005)

A new study has opened the way for detailed imaging of neocortical activity at the single-cell level. Kerr et al. used in vivo two-photon calcium imaging of bulk-labelled tissue in the rat neocortex, and observed strong calcium signals in axonal structures, which are indicative of input activity, and local spike patterns, which reflect output activity. Using these measures, they found that spontaneous neocortical activity is sparse and heterogeneously distributed across neuronal populations.

\section{MEMORY}

Linking new information to a reactivated memory requires consolidation and not reconsolidation mechanisms.

Tronel, S. et al. PLoS Biol. 3, e293 (2005)

New memories become stabilized by the process of consolidation. But they can become labile again if they are reactivated by recall, and require another phase of RNA and protein synthesis to be maintained. This process of reconsolidation was a strong candidate for linking new information to reactivated memories. Surprisingly, Tronel et al. show that reconsolidation does not contribute to this process. Instead, molecular mechanisms similar to those underlying consolidation are used to form an association between new and reactivated information.

\section{SYNAPTIC TRANSMISSION}

\section{Heterogeneity in synaptic transmission along a} Drosophila larval motor axon.

Guerrero, G. et al. Nature Neurosci. 8, 1188-1196 (2005)

To address the question of how transmission strength is distributed among the many connections between a motor neuron and its muscle partner, Guerrero et al. created synapcam, a version of the $\mathrm{Ca}^{2+}$ reporter cameleon. Synapcam can detect $\mathrm{Ca}^{2+}$ influx through postsynaptic glutamate receptors with single-impulse and single-bouton resolution. This approach was combined with presynaptic imaging to show that transmission strength is determined mainly presynaptically, varying in a gradient along the length of axonal branches, with distal connections making stronger functional associations.

\section{VISION}

Urochordate $\beta \gamma$-crystallin and the evolutionary origin of the vertebrate eye lens.

Shimeld, S. M. et al. Curr. Biol. 15, 1684-1689 (2005)

The formation of the eye lens and the high concentration of crystallins expressed by lens cells are crucial for the ability of the eye to form a clear, focused image on the retina, which is unique to vertebrates. Shimeld and colleagues have now identified a gene in the primitive visual system of the vertebrates' nearest relative — the sea squirt — that is highly homologous to $\beta \gamma$-crystallin. This suggests that the vertebrate $\beta \gamma$-crystallin gene might have evolved from its counterpart in sea squirts. 